Www.jmscr.igmpublication.org

Index Copernicus Value: 79.54

ISSN (e)-2347-176x ISSN (p) 2455-0450

crossref DOI: https://dx.doi.org/10.18535/jmscr/v7i6.37

Journal Of Medical Science And Clinical Research

\title{
Management of CBD Stones - Our Experience
}

\author{
Authors \\ Dr Kodanda Ramu Ulala ${ }^{1}$, Dr Munagavalasa Aditya Ram², \\ Dr Konkena Janardhana Rao ${ }^{3 *}$, Dr Sekuboyina Kiran Datta ${ }^{4}$ \\ ${ }^{1}$ Assistant Professor of Surgery, Andhra Medical College, Visakhapatnam, India \\ ${ }^{2,4}$ Junior Resident of Surgery, Andhra Medical College, Visakhapatnam, India \\ ${ }^{3}$ Associate Professor of Surgery, Government Medical College (RIMS), Cuddapah, India \\ *Corresponding Author \\ Dr Konkena Janardhana Rao \\ Associate Professor of Surgery, Government Medical College (RIMS), Cuddapah, India
}

\begin{abstract}
Background: Choledocholithiasis occurs in approximately $20 \%$ of patients with cholelithiasis. A majority of stones form in the gallbladder and then pass into the common bile duct, where they may remain asymptomatic or generate symptoms like biliary colic, jaundice, cholangitis, pancreatitis. Confirmatory diagnosis is made with imaging which includes ultrasonogram, Magnetic resonance cholangiopancreatography (MRCP) and Endoscopic retrograde cholangiopancreatography (ERCP). Treatment varies locally; however, ERCP with sphincterotomy is most commonly employed with a high degree of success. Difficult anatomy and difficult stone burden require surgical CBD exploration. Knowledge of these treatment strategies will optimize outcomes. The aim of this study is to explore and assess various treatment strategies employed in cases of choledocholithiasis in our setup and to determine their outcome.

Materials \& Methods: A retrospective database containing 106 cases with choledocholithiasis from June 2016 to March 2018 were evaluated. All the patients underwent ultrasonography (USG), MRCP and had their liver function tests estimated. ERCP was done when necessary. Other causes of obstructive jaundice like benign or malignant strictures, periampullary carcinomas, post cholecystectomy strictures were excluded from the study.

Results: Of the 106 patients in this study, 57.5\% were men and $42.5 \%$ were women. The mean age was 48.7 years (range 40-60 years). A total of 30 (28.3\%) patients underwent ERCP guided stenting along with stone withdrawal and sphincteroplasty, followed by Laparoscopic cholecystectomy. 45 (42.45\%) patients had failed stone withdrawal for whom biliary stenting was done. These patients underwent open cholecystectomy and CBD exploration with primary repair. 28 (26.41\%) cases with stone size more than $15 \mathrm{~mm}$ directly underwent open cholecystectomy and CBD exploration with T-tube drainage. Average size of the stone for which extraction via ERCP was done is $11.8 \mathrm{~mm}$. One case of recurrent choledocholithiasis was managed with Roux-en-Y hepaticojejunostomy. Two cases of choledocholithiasis with markedly dilated CBD were managed with choledochoduodenostomy.

Conclusion: The management of choledocholithiasis has reached a point in its evolution where more options are available than some institutions can support. The data currently available support a wide range of satisfactory treatment algorithms. Treatment may be endoscopic, percutaneous, open, or laparoscopic. Given the multiple alternatives available, sometimes it is difficult to decide on the right one for a particular patient. Frequently, the best path is the one the surgeon is most adept at or the one that local expertise can accomplish most safely. A two-staged procedure consisting of ERCP, followed by laparoscopic cholecystectomy, should be the first line of treatment for common bile duct (CBD) stones. In cases where ERCP fails or stone size is larger, open surgical procedures still remain a relevant and a definitive option in resource-constrained setups.

Keywords: Choledocholithiasis-endoscopic retrograde cholangiopancreatography-cholelithiasis-surgery.
\end{abstract}




\section{Introduction}

The presence of a stone or stones within the common bile duct (CBD) is known as choledocholithiasis. Choledocholithiasis is reported in 3 to $22 \%$ of cholecystectomies ${ }^{[1,2]}$. Choledocholithiasis is classified as primary or secondary according to stone origin ${ }^{[3]}$. Primary choledocholithiasis refers to stones formed directly within the biliary tree, while secondary choledocholithiasis refers to stones ejected from the gallbladder.

Currently, laparoscopic cholecystectomy (LC) is the standard surgical treatment for cholelithiasis. Because manipulation of common bile duct stones (CBDS) during LC is not a common practice, preoperative assessment and endoscopic removal seems to be the most proper way to manage CBDS. In distinction to cholelithiasis, the majority of choledocholithiasis is symptomaticspecifically, right upper quadrant pain, caused by distention of the extrahepatic bile duct, along with nausea and vomiting ${ }^{[4]}$. Management of CBDS usually requires two separate teams: the medical gastroenterologist and the surgical team ${ }^{[5]}$. The main options for treatment are pre or postoperative ERCP with endoscopic biliary sphincterotomy (EST), laparoscopic or open surgical bile duct clearance. Laparoscopic bile duct clearance is not available in our setup. Nonsurgical options like electrohydraulic lithotripsy (EHL), extracorporeal shockwave lithotripsy (ESWL), dissolving solutions, and laser lithotripsy are available in some setups. It is unlikely that one option will be appropriate for all clinical circumstances in all centres. Variables such as disease status, patient demographics, availability of endoscopic, radiological and surgical expertise, and healthcare economics will all have significant influence on practice ${ }^{[6]}$.

\section{Materials and Methods}

A retrospective study containing 106 cases with choledocholithiasis from June 2016 to March 2018 were evaluated. All the patients underwent ultrasonography (USG), MRCP and had their liver function tests estimated. ERCP was done when necessary. The following liver function test results were accepted as abnormal: total bilirubin greater than $1.17 \mathrm{mg} / \mathrm{dl}$, direct bilirubin greater than 0.35 $\mathrm{mg} / \mathrm{dl}$, alkaline phosphatase (ALP) greater than 279 units/l, alanine amino transferase (ALT) greater than 40 units/l, and aspartate amino transferase (AST) greater than 37 units/l. Some parameters such as abdominal USG findings, liver function tests, age, gender, resolved pancreatitis and acute cholecystitis were studied.

This study serves to delineate the current relevant concepts in the varying treatments of patients that present with CBDS. Those patients who had successful ERCP underwent laparoscopic or open cholecystectomy during the same admission. On the other hand, in patients whom ERCP failed, they underwent either choledochotomy (with or without T-tube) or biliary-enteric drainage procedures like choledochoduodenostomy or choledochojejunostomy.

\section{Results}

Of the 106 patients in this study, $57.5 \%$ were men and $42.5 \%$ were women. The mean age was 48.5 years (range, 40-60years).

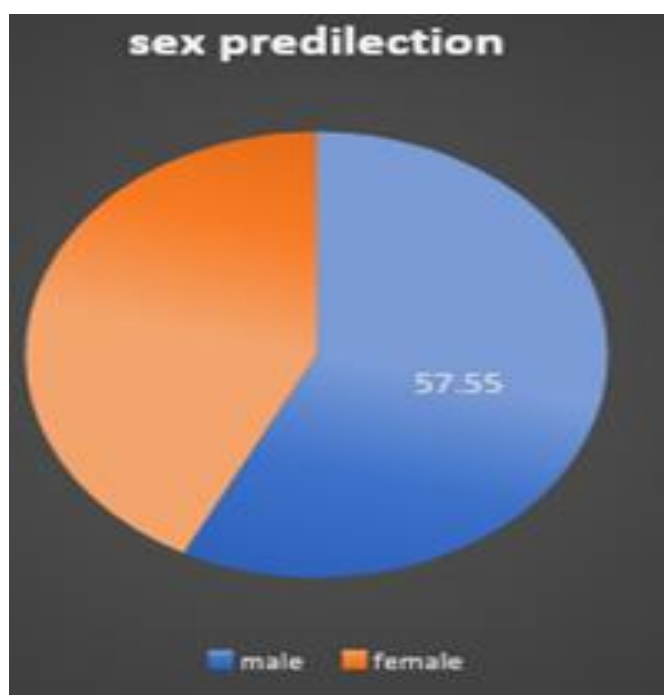




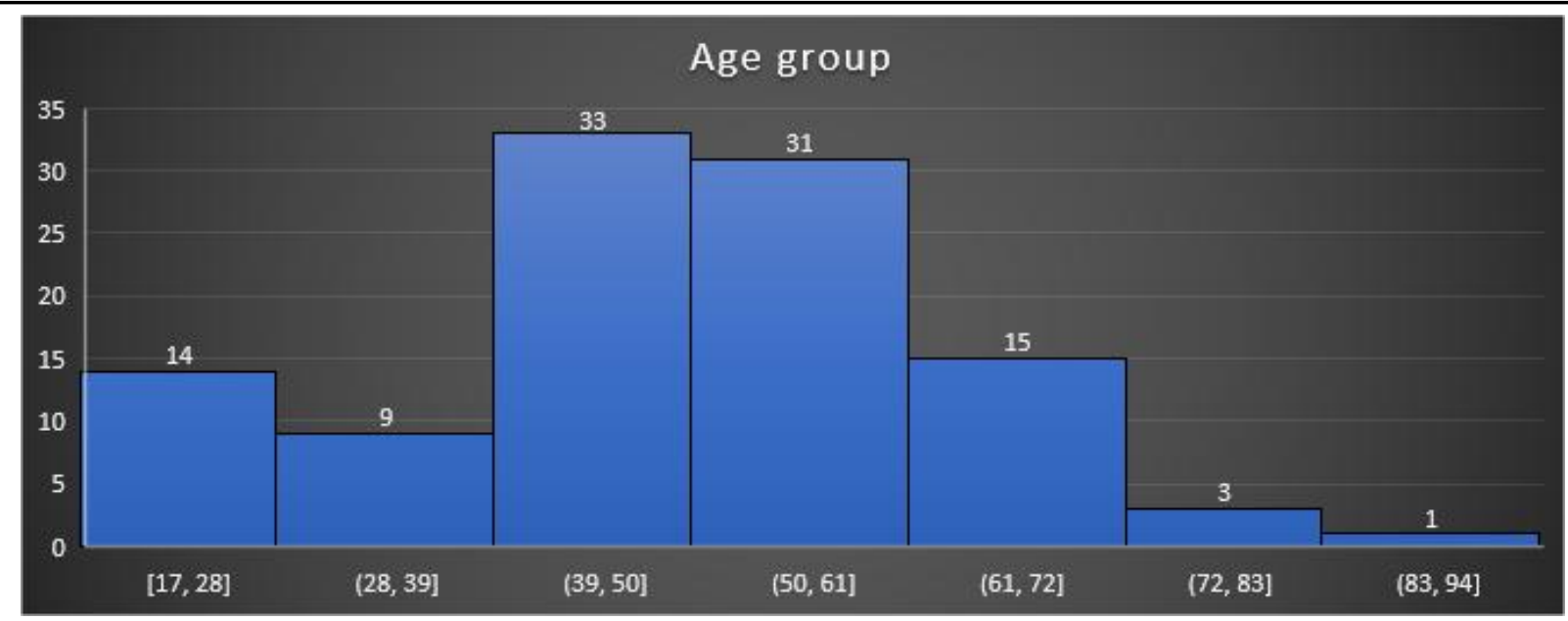

A total of 30 (28.3\%) patients underwent ERCP guided stenting along with stone withdrawal and sphincteroplasty, followed by Laparoscopic cholecystectomy later. 45 (42.4\%) patients had failed stone withdrawal (stenting done). These patients underwent open cholecystectomy and CBD exploration with primary repair. 28 $(26.41 \%)$ cases with stone size more than $15 \mathrm{~mm}$ directly underwent open cholecystectomy and CBD exploration with T-tube drainage. Average size of the stone below which balloon sweeping or withdrawal was done is $11.9 \mathrm{~mm}$. One case of recurrent choledocholithiasis was managed with Roux-en-Y hepaticojejunostomy. Two cases of choledocholithiasis with markedly dilated CBD managed with choledochoduodenostomy.
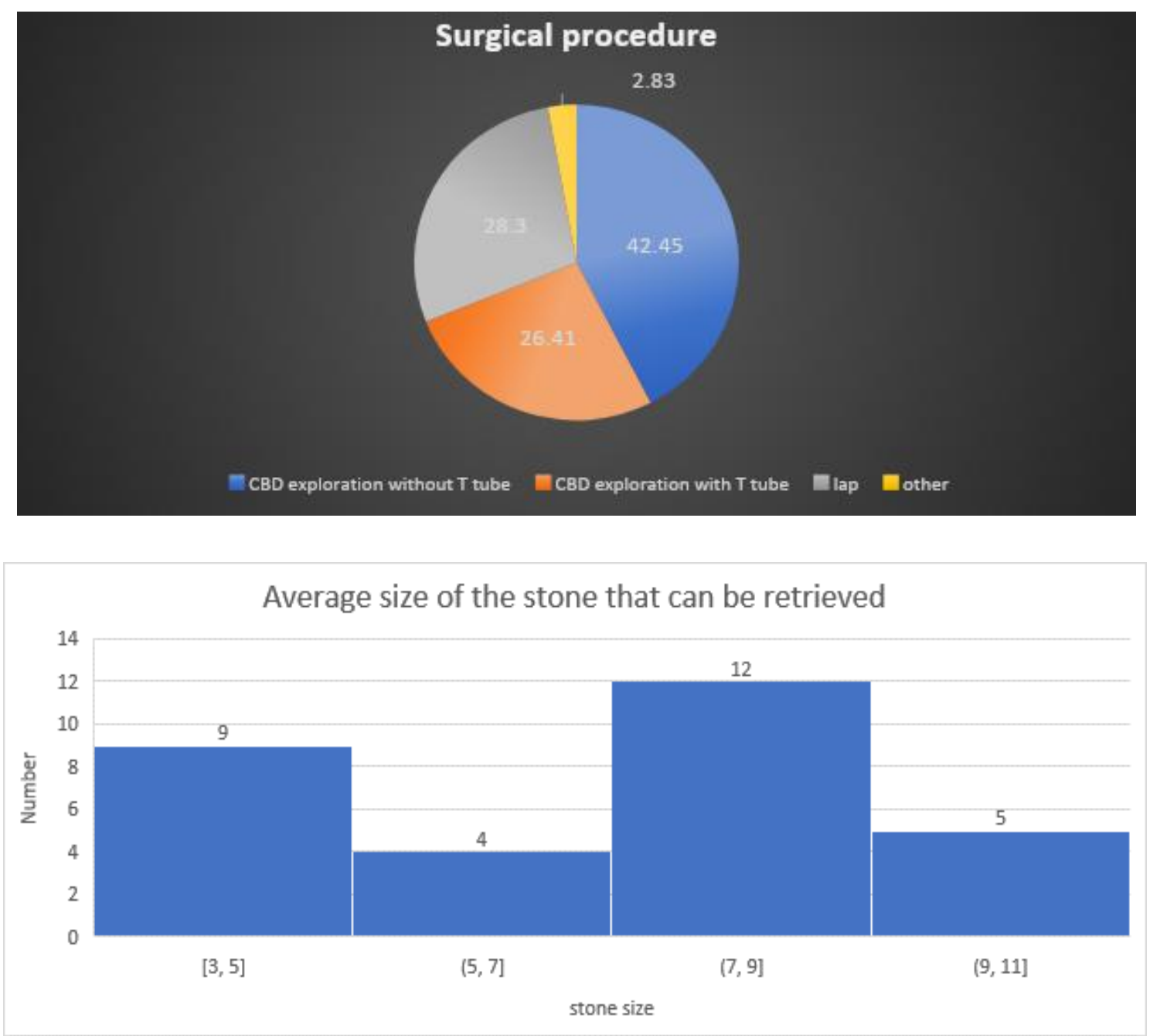


\section{Discussion}

In our study, choledocholithiasis coexists in $18 \%$ of patients presenting with cholelithiasis which correlates with the literature 10-18\% (Dasari et al, 2013). The majority of enrolled patients in our study were male $(57.55 \%)$. This cannot be explained by the fact that gallstone diseases, including choledocholithiasis, are more common in females all over the world. ERCP has been accepted internationally as a first-line treatment option for choledocholithiasis with a success rate of almost $73 \%$. In our study, $98.58 \%$ of patients underwent ERCP. Successful stone retraction correlated well with the stone size and location of the stone in CBD rather than the number of stones. In our study, only $28.3 \%$ of cases were successfully managed with ERCP guided stone withdrawl. In our study, $53.06 \%$ of stones below $10 \mathrm{~mm}$ were successfully retrieved out of which most were located in distal CBD. $16.98 \%$ cases there is a failure of ERCP mediated withdrawl and stenting. Rest of the cases were stented. $6 \%$ of patients suffered from post ERCP pancreatitis which were manged conservatively. This is much lower than the figure quoted in international studies $^{[7]}$. Large stones, over $15 \mathrm{~mm}$, were directly posted for CBD exploration due to lack of reliable mechanical lithotripter and other alternatives at the time of study. Surgical clearance of the CBD, once a common procedure, is now only considered when ERCP fails. Currently, the only surgical procedure which can rival ERCP in terms of cost, as well as efficacy, is a single-staged laparoscopic procedure consisting of both cholecystectomy and CBD exploration ${ }^{[8-9]}$. This, unfortunately, could not be evaluated in our setup due to lack of advanced laparoscopic equipment and training.

\section{Conclusion}

The management of choledocholithiasis has reached a point in its evolution where more options are available than some institutions can support. The data currently available support a wide range of satisfactory treatment algorithms.
Open common bile duct exploration and stone extraction have enjoyed satisfactory results for decades. The advances in laparoscopic technology for the general surgeon that have been developed over the last 10 to 15 years have created a seductive atmosphere for consideration in the management of choledocholithiasis. Newer techniques have the drawback of being equipment-dependent and require advanced and continued training in a field that in constantly in flux. Laparoscopic management of CBDS remains technically challenging for surgeons and financially challenging for institutions. A twostaged procedure consisting of ERCP, followed by laparoscopic cholecystectomy, should be the first line of treatment for common bile duct (CBD) stones where resources are constrained. In cases where ERCP fails or stone size is larger, open surgical procedures still remain a relevant and a definitive option. Institutions that cannot or choose not to bear the expense of equipment acquisition or the personnel to support and operate this equipment must follow different guidelines than institutions that do have access to such capabilities.

\section{References}

1. Williams EJ, Green J, Beckingham I, Parks R, Martin D, Lombard M; British Society of Gastroenterology. Guidelines on the management of common bile duct stones (CBDS). Gut 2008;57(7): 10041021

2. Gurusamy KS, Giljaca V, Takwoingi Y, et al. Ultrasound versus liver function tests for diagnosis of common bile duct stones. Cochrane Database Syst Rev 2015;2(2):CD011548

3. European Association for the Study of the Liver (EASL). Electronic address: easloffice@easloffice.eu. EASL Clinical Practice Guidelines on the prevention, diagnosis and treatment of gallstones. J Hepatol 2016;65(1):146-181 
4. European Association for the Study of the Liver (EASL). Electronic address: easloffice@easloffice.eu. EASL Clinical Practice Guidelines on the prevention, diagnosis and treatment of gallstones. J Hepatol 2016;65(1):146-181

5. J. P. Dorman, M. E. Franklin Jr., and J. L. Glass, "Laparoscopic common bile duct exploration by choledochotomy: an effective and efficient method of treatment of choledocholithiasis," Surgical Endoscopy, vol. 12, no. 7, pp. 926-928, 1998.

6. D. L. Carr-Locke, "Cholelithiasis plus choledocholithiasis: ERCP first, what next?" Gastroenterology, vol. 130, no. 1, pp. 270-272, 2006

7. Elmunzer BJ: Reducing the risk of postendoscopic retrograde cholangiopancreatography pancreatitis. Dig Endosc. 2017

8. Bansal VK, Krishna A, Rajan K, et al.: Outcomes of laparoscopic common bile duct exploration after failed endoscopic retrograde cholangiopancreatography in patients with concomitant gall stones and common bile duct stones: a prospective study. J Laparoendosc Adv Surg Tech A. 2016, 26:985-91.

9. Ding G, Cai W, Qin M: Single-stage vs. two-stage management for concomitant gallstones and common bile duct stones: a prospective randomized trial with longterm follow-up. J Gastrointest Surg. 2014, 18:947-51. 